\title{
Parámetros genéticos de la prolificidad y la supervivencia hasta el sacrificio en corderos de raza Merina
}

\author{
Menéndez-Buxadera, A. '; Medina, C. ${ }^{\text {; }}$ Gómez, J. '; Barajas, F. y Molina, A. ${ }^{1}$ \\ 'Grupo Meragem. Departamento de Genética. Facultad de Veterinaria. Universidad de Córdoba. España. \\ ${ }^{2}$ Asociación Nacional de Criadores de Ganado Ovino de raza Merina. Madrid. España.
}

\section{PALABRAS CLAVE ADICIONALES}

Ovino.

\section{Heredabilidad.}

Modelo umbral.

Selección animal.

Rendimiento reproductivo.

\section{RESUMEN}

Utilizando los datos del núcleo de control cárnico de la raza Merina, se ha realizado un análisis genético de la prolificidad (entendida como medida indirecta de la supervivencia de las crías hasta el nacimiento), y de la supervivencia de los corderos desde el nacimiento al final de cebo. Se realizó un análisis univariado, con un modelo umbral para la prolificidad y un modelo animal con metodología REML para la supervivencia hasta el final del cebo. La estimación de los parámetros genéticos determinó una $h^{2}$ de la prolificidad de $0,115(0,166$ y 0,171 para los efectos genéticos directos y maternos respectivamente) y de 0,072 para la supervivencia postnatal $(0,176$ para efectos genéticos directos y 0,040 para los efectos maternos), siendo la correlación genética entre ambos caracteres de - 0,044 para los efectos genéticos directos y de -0,201 para los efectos genéticos maternos. Estas $h^{2}$ son suficientemente elevadas para asegurar una respuesta positiva a la selección, tanto para prolificidad, como para supervivencia hasta el final del cebo. No obstante, dada la correlación genética negativa entre ambos tipos de caracteres, la selección para la mejora de la prolificidad debe llevar acarreada una mejora ambiental y genética de la capacidad para la supervivencia de los corderos si no se quiere anular el crecimiento ponderal de corderos nacidos con un incremento de su mortalidad.

\section{Genetic parameters of the prolificacy and survival to the slaughterhouse in Merina sheep lamb}

\section{SUMMARY}

\section{ADDITIONAL KEYWORDS}

Sheep.

Heritability.

Threshold model.

Animal selection.

Reproductive performance.

\section{INFORMACIÓN}

\section{Cronología del artículo.}

Recibido/received: 25.04 .15

Aceptado/Accept: 26.02.16

Online: 15.09 .16

Correspondencia a los autores/Contact e-mail:

gelmoala@uco.es

\section{INTRODUCCIÓN}

En los últimos años, dentro de los objetivos de selección de la raza Merina (Magrama, 2011), ha ido cobrando cada vez más importancia la productividad total del
It was conducted a genetic analysis of prolificacy and survival of the Merina breed lambs from birth to end of fattening. Univariate analysis, with a threshold model for prolificacy, and an animal model with REML methodology, for survival at the end of fattening, was performed. The estimation of genetic parameters determined a prolificacy heritability $\left(h^{2)}\right.$ equal to 0.115 $(0.166$ and 0.171 for direct genetic effects and maternal effects, respectively) and equal to 0.072 for postnatal survival ( 0.176 for direct genetic effects and 0.040 for maternal effects). Additionally, the genetic correlation between the two traits was -0.044 and -0.201 , for direct and maternal genetic effects. These $h^{2}$ are high enough to ensure a positive response to selection for both prolificacy and survival until the end of fattening. However, given the negative genetic correlation between the two types of traits, selection for improved prolificacy should be accompanied both for environmental and genetic improvement of the lambs survival capacity, if you do not want to penalize the lambs weight growth born with increased mortality. rebaño, en el que intervienen los relacionados con el crecimiento, la fertilidad, la prolificidad (o tamaño de camada, TC) y la supervivencia de los corderos. Existen suficientes antecedentes que indican que, en los medios difíciles en los que se explota la raza Merina, existe una 
relación directa entre prolificidad y supervivencia. Los estudios realizados en esta misma raza (Molina et al., 2015) muestran que, al menos fenotípicamente, un incremento de la prolificidad siempre va acompañada de un incremento de la mortalidad de los corderos, si no se tienen en cuenta medidas correctoras (que en muchos casos pueden ser económicamente prohibitivas). Por lo tanto, si se pretende realizar una mejora genética de la prolificidad, es necesario no sólo estimar la importancia del componente genético sobre dicho carácter, sino la magnitud de la posible correlación genética existente entre la prolificidad y la mortalidad (o su inversa, la tasa de supervivencia) de los corderos. Si es elevada, la respuesta indirecta correlacionada sobre dicha mortalidad puede hacer desaconsejar dicha selección. Así mismo, la estimación de la heredabilidad $\left(\mathrm{h}^{2}\right)$ del carácter supervivencia del cordero permitirá estimar la viabilidad de una selección para la supervivencia que permita atenuar este problema desde el punto de vista genético.

\section{MATERIAL Y MÉTODOS}

Para obtener los parámetros genéticos del TC (entendido como medida indirecta de la supervivencia de las crías hasta el nacimiento) y supervivencia de la raza Merina, se analizaron los 55439 partos de 32556 ovejas, procedentes de 71 ganaderías pertenecientes al Esquema de Selección. Los 65977 corderos nacidos de estos partos fueron controlados hasta el sacrificio, existiendo información quincenal del cordero (vivo/muerto), desde el nacimiento hasta el final del cebo. Se realizó un análisis univariado, con un modelo umbral (Foulley y Manfredi, 1991) para TC y un modelo animal con metodología REML para la supervivencia (en este caso, se analiza el tiempo que permanece en la explotación hasta que muere). En ambos modelos, se incluyeron como efectos fijos la ganadería-año-mes del parto, el sexo del cordero y la edad al parto de la oveja (covariable lineal). Para la variable supervivencia, se incluyó el factor tamaño de la camada-sexo, en lugar del sexo. Ambos modelos incluyeron como efectos aleatorios el efecto genético directo del cordero, el efecto materno (efecto de la oveja) y el efecto ambiental permanente de la oveja. Un segundo modelo bivariado fue utilizado para estimar la correlación genética entre prolificidad y supervivencia, en el que se eliminó, como factor del modelo para esta última, la prolificidad. Para ello se utilizó el software Asreml 3 (Gilmour et al., 2009).

\section{RESULTADOS Y DISCUSIÓN}

Los resultados muestran una prolificidad media de 1,19. En la figura 1 se presenta la evolución de la media mínimo-cuadrática de la prolificidad en función de la edad a la cubrición de la oveja.

Se puede observar (figura 2) como la prolificidad es más elevada entre los 3,5 y 8 años de edad de la reproductora, con acumulación de una mayor probabilidad de presentar un parto doble (entre los 3 y los 6,5 años de edad) o de un parto triple (4 y los 6,5 años) .

El análisis por ganaderías demuestra una amplia

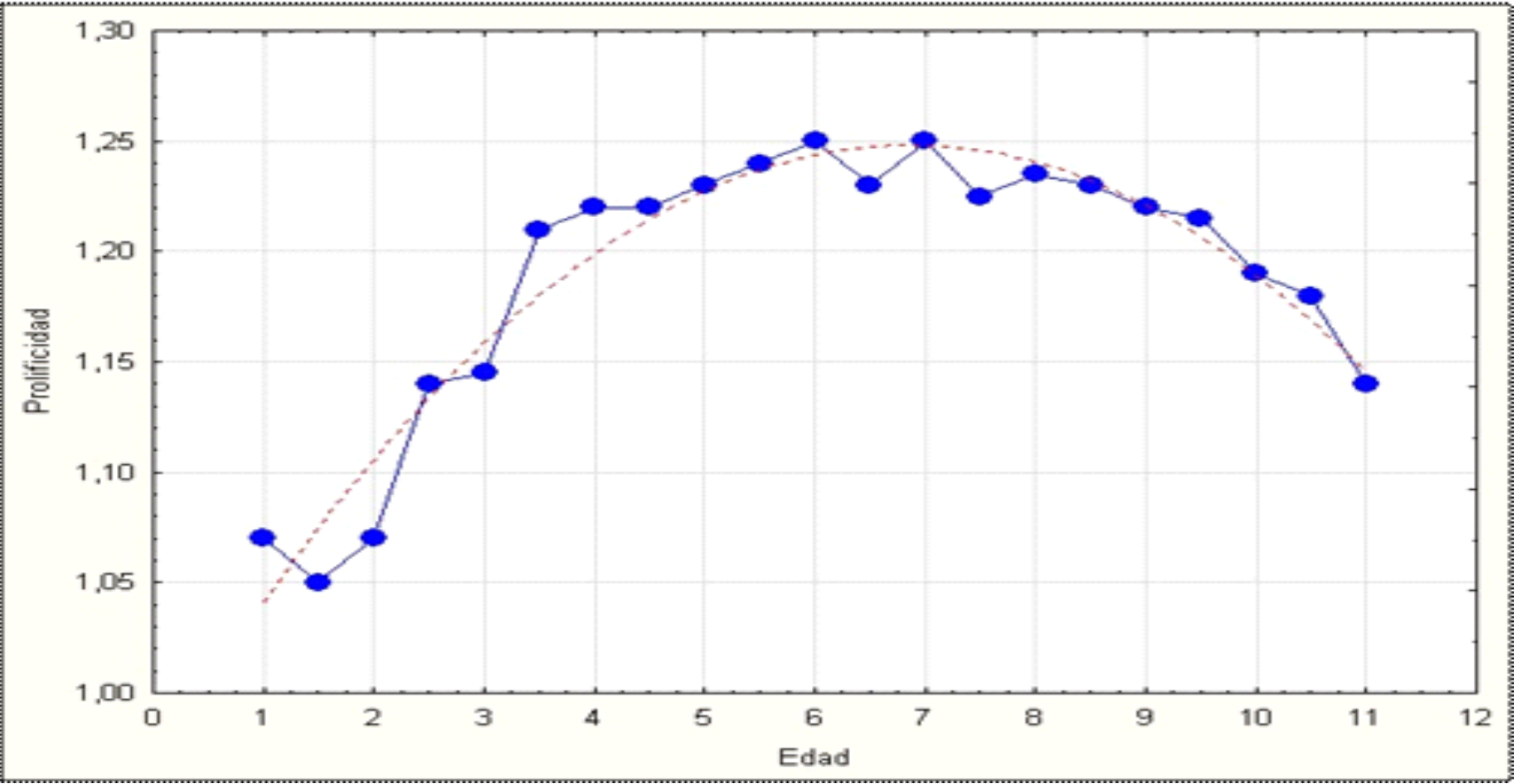

Figura 1. Evolución de la tasa de prolificidad en función de la edad de la oveja Merina (Evolution of prolificity as a function of age in Spanish Merino sheep). 


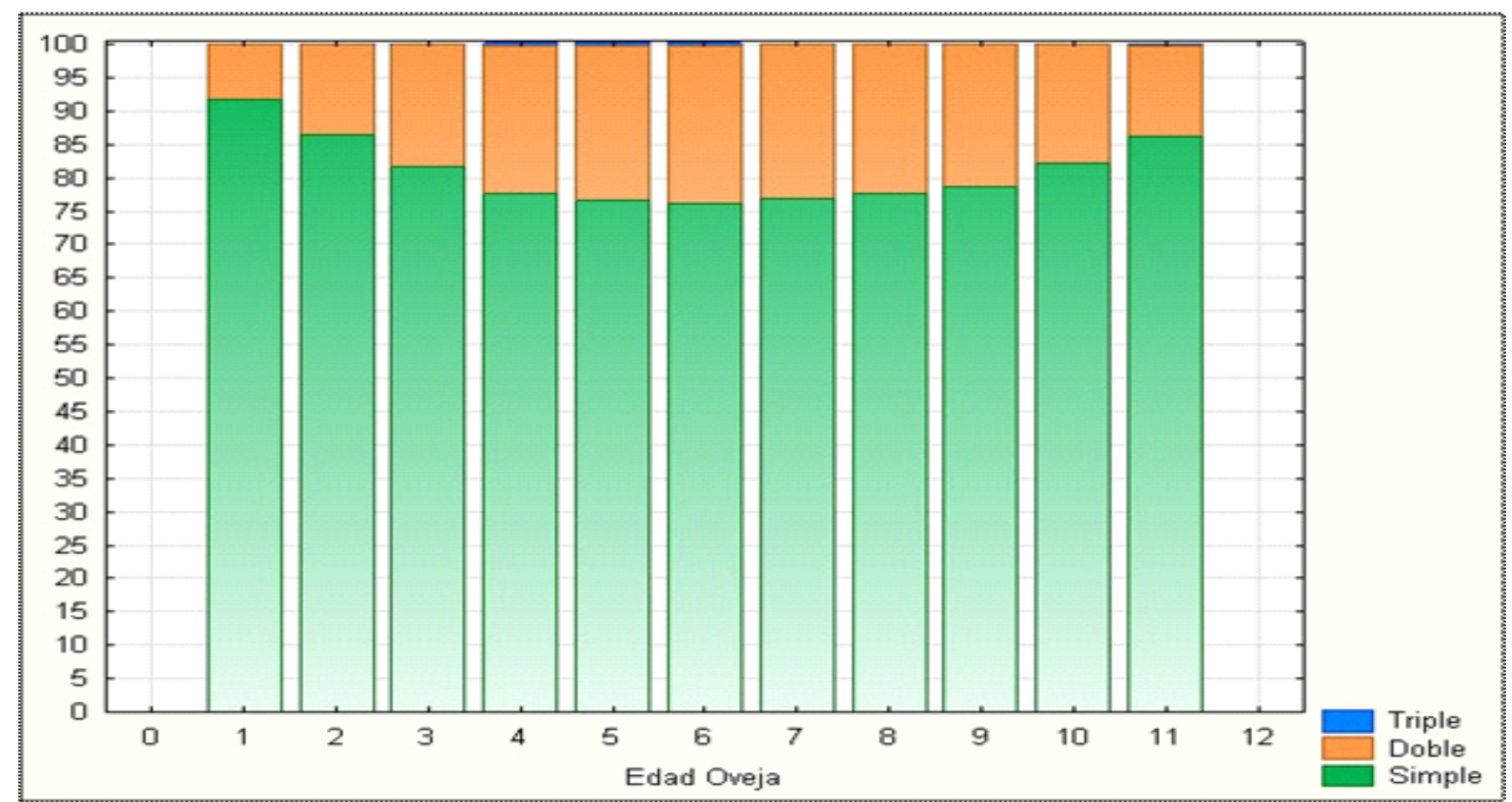

Figura 2. Porcentaje de partos simples, dobles y triples en función de la edad de la oveja (Distribution (\%) of lambing with single, double and triplet lambs as a function of age in Spanish Merino sheep).

variabilidad, existiendo explotaciones con prolificidades medias muy próximas a 1, y otras próximas a 1,40 (figura 3).
No obstante, dado el difícil medio en que se explota el ovino en el sudoeste español, el incremento de la prolificidad lleva aparejada un incremento de la morta-

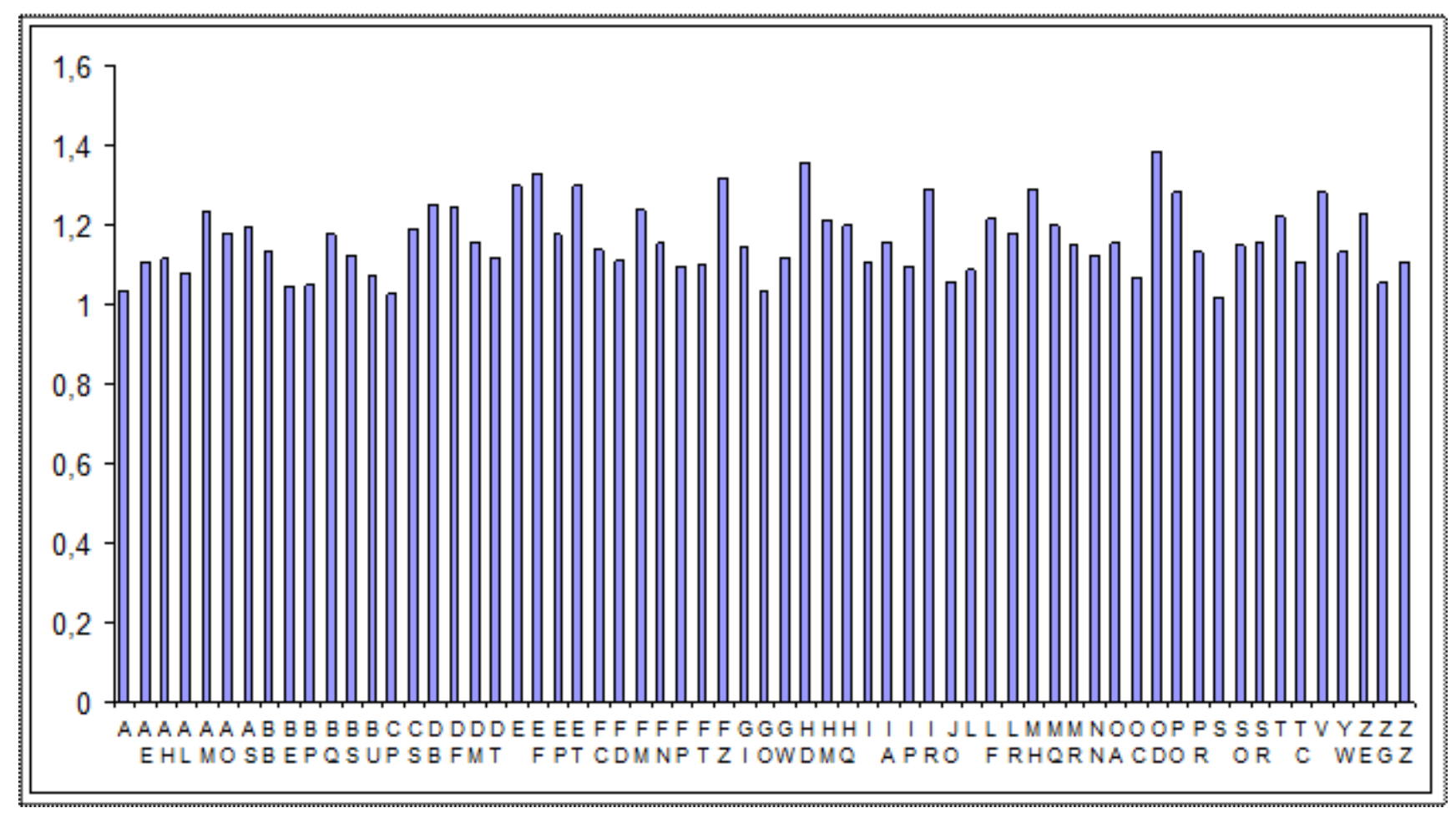

Figura 3. Promedio de prolificidad de ganaderías de raza Merina (Average in prolificity in the Spanish Merino flocks). 
lidad principalmente en las dos primeras semanas de vida del cordero. En el análisis preliminar de los corderos controlados desde el nacimiento hasta el final del cebo, se ha determinado una mortalidad global del $8,19 \%$, distribuida según se muestra en la figura 4. Se puede observar una supervivencia global al nacimiento del 98,9\%, del 93,76 al destete (mortalidad en el periodo predestete del $6,20 \%$ ) y del $91,81 \%$ al final del cebo (mortalidad en el periodo de cebo del $0,91 \%)$.

Esta misma variabilidad (que conlleva también posibilidades de mejora) se puede observar en el caso de la tasa de mortali$\mathrm{dad} /$ supervivencia en la figura 5 . Se puede observar la existencia de grandes diferencias entre ganaderías.

La mayoría de los estudios publicados sobre TC (ver la revisión de MenendezBuxadera et al., 2004), lo considera un rasgo que depende fundamentalmente del genotipo de la madre. No obstante, existen evidencias científicas (Asworth, 1992; Gill et al., 1998; Menendez-Buxadera et al., 2004) de que el TC es el resultado de la ac-

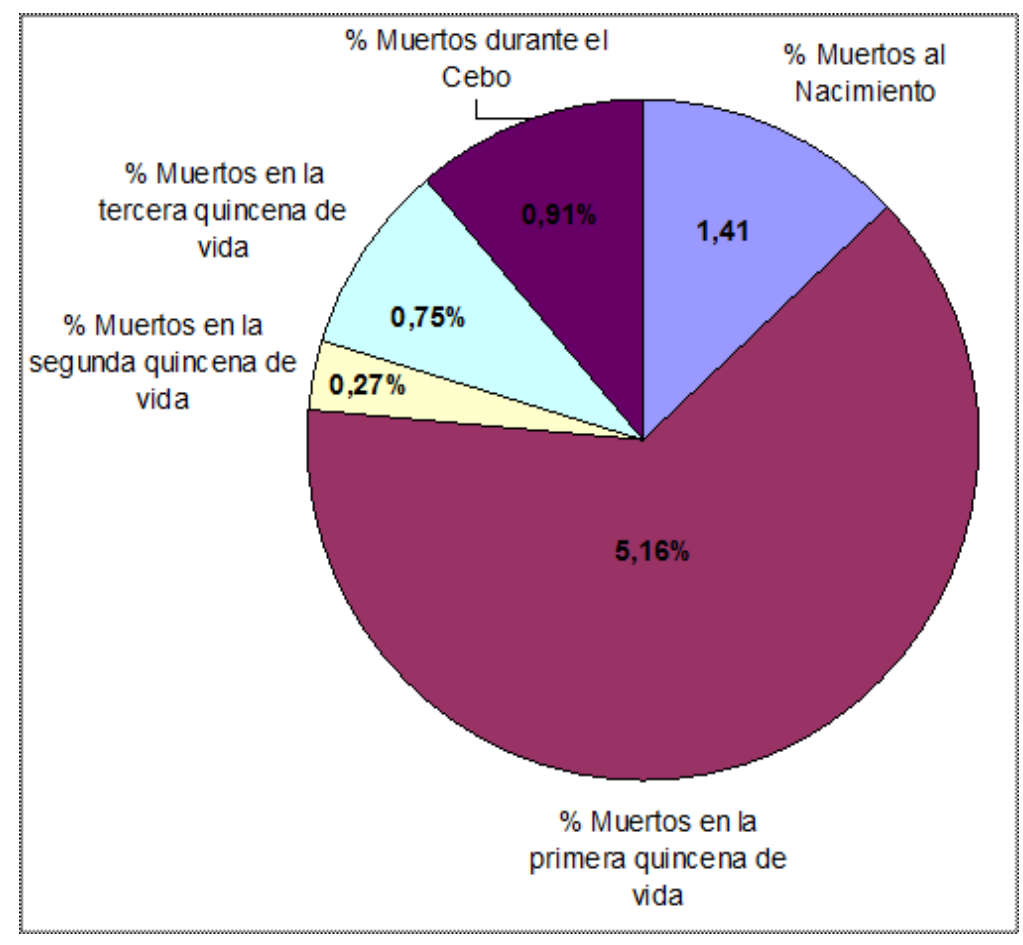

Figura 4. \% Mortalidad de los corderos Merinos desde el nacimiento hasta el sacrificio (Mortality (\%) of lambs from birth to slaughter in Spanish Merino sheep). ción e interacción (antagónica, incluso), de factores ligados a más de un componente genético (especialmente oveja y crías), de modo similar a lo que ocurre con la dificultad al parto del ganado (figura 6).

La metodología más adecuada para la estimación de parámetros en este carácter suele ser el modelo umbral (threshold model), especialmente en aquellas especies

como la ovina donde el número de crías no puede considerarse en ningún caso un carácter normal (que siga la distribución normal de Gauss), aunque también se pueden encontrar algunas estimaciones mediante modelos REML (especialmente en cerdos, dada la elevada prolificidad que presenta). Utilizando un modelo umbral que incluía ambos efectos (el efecto directo del pro-

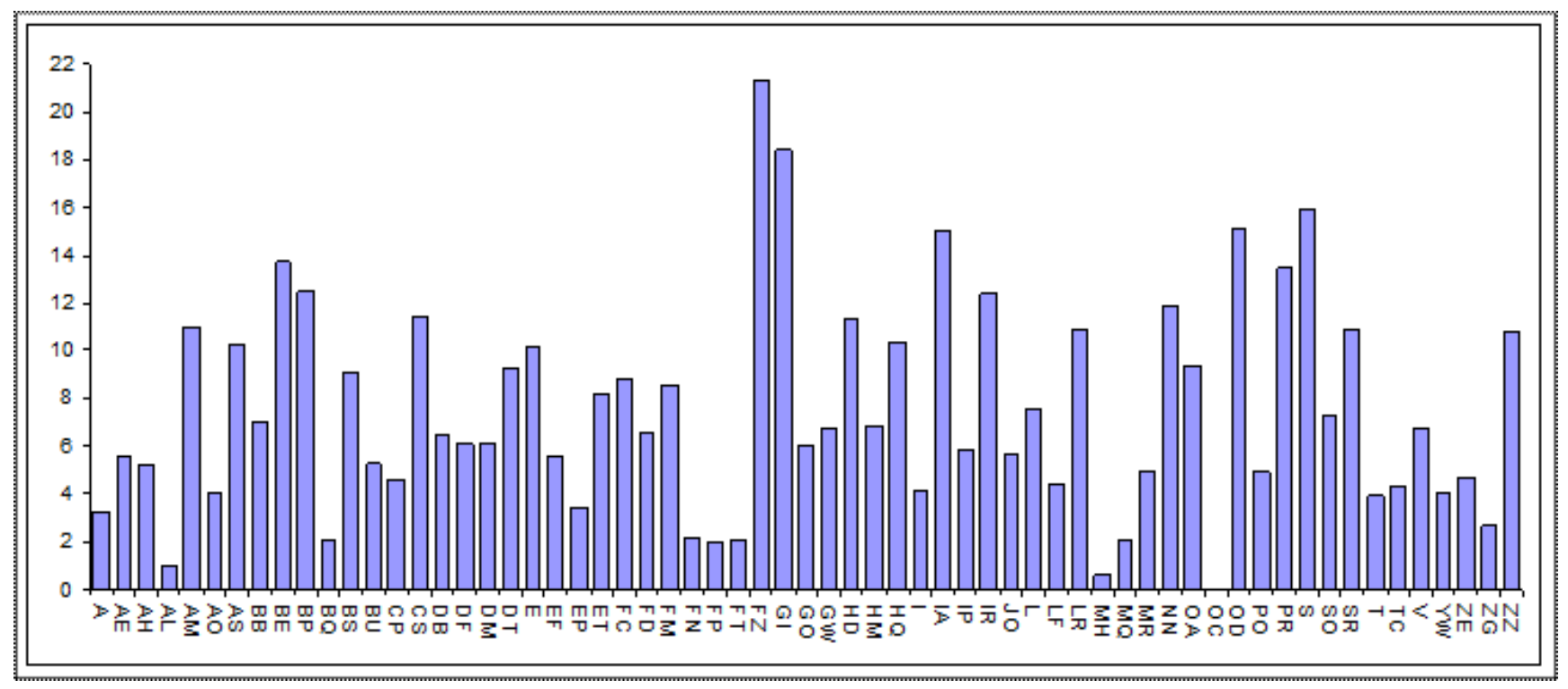

Figura 5. Promedio de mortalidad (\%) al final del cebo de ganaderías de raza Merina (Average mortality (\%) in the Spanish Merino flocks). 


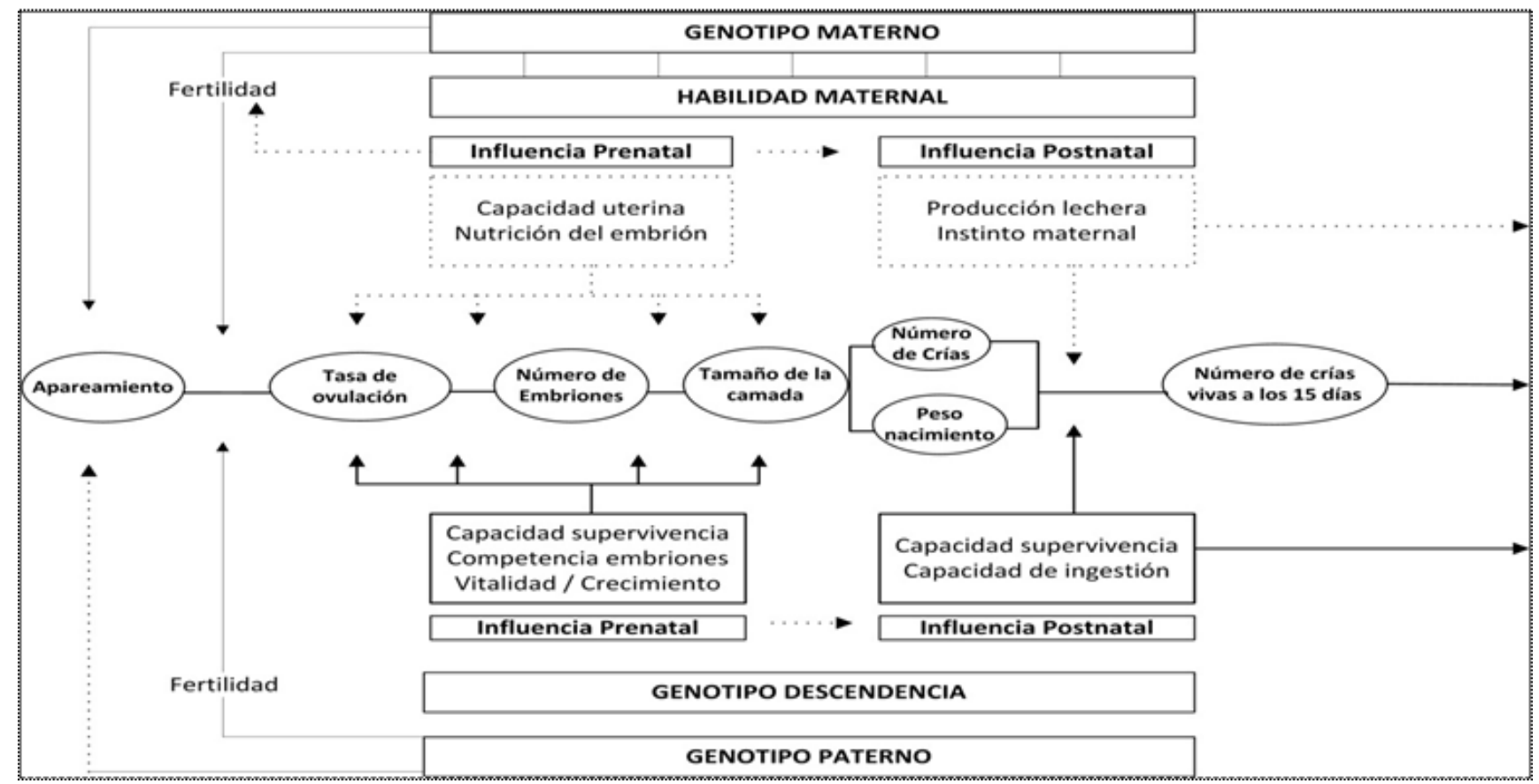

Figura 6. Elementos incluídos en la productividad del rebaño de pequeños rumiantes antes del destete con énfasis en la contribucion de padres y madres (las condiciones de manejo y gestión general no se presentan) (Elements included in small ruminant flock productivity before weaning with emphasis on the contributions of the sire and dam (general management and husbandry conditions are not presented). (Adaptado de Menendez-Buxadera et al., 2004).

pio cordero y el de la madre), se obtuvieron los parámetros que se resumen en la tabla I. Así, la h² para TC es de 0,115 , suficientemente alta para poder obtener respuesta a la selección para este carácter. Las $\mathrm{h}^{2}$ de los componentes directos (del cordero) y materno (de la oveja) son de mediana magnitud, algo superior en elcaso del efecto de la madre, pero presentan una correlación genética negativa.

En el caso de los parámetros genéticos para la supervivencia hasta el final del cebo, destaca la magnitud de los efectos directos (debidos al propio cordero) sobre la capacidad de supervivencia al final del cebo, muy superior a la $\mathrm{h}^{2}$ para los efectos maternos.

No obstante, la elevada correlación genética negativa entre efectos genéticos directos y maternos obliga a analizar más en profundidad la estrategia de selección para este carácter, para el que una selección clásica determinaría una limitada mejora genética global en la población.

Finalmente, la correlación genética entre ambos tipos de caracteres (tamaño de camada y supervivencia al cebo) mostró valores negativos entre efectos genéticos directos, lo mismo que entre efectos maternos (aunque, en el primer caso, muy cercanos a cero): una mayor prolificidad va a determinar una menor supervivencia. No obstante, la moderada magnitud hace prever un cierto margen de mejora de ambos caracteres de forma conjunta.

\section{CONCLUSIONES}

En los últimos años, muchos criadores de ovejas merinas demandan una mejora de otros caracteres con

Tabla I. Parámetros genéticos del carácter prolificidad en la raza Merina (Genetic parameters for prolificity in Merina breed).

\begin{tabular}{lc}
\hline Parámetro & Prolificidad \\
\hline Heredabilidad para los efectos directos & 0,166 \\
Heredabilidad para los efectos maternos & 0,171 \\
Correlación genética efectos directos-maternos & $-0,538$ \\
Heredabilidad total & 0,115 \\
Correlación TC- Supervivencia efectos directos & $-0,044$ \\
Correlación TC- Supervivencia efectos maternos & $-0,979$ \\
Correlación TC- Supervivencia directos-maternos & $-0,201$ \\
\hline
\end{tabular}


una clara repercusión económica, tanto para los criadores en pureza como para los que realizan el cruce industrial. Dentro de estos parámetros, destaca la prolificidad, uno de los más modestos de esta raza, con niveles medios en torno a $120 \%$.

Los parámetros genéticos hallados han confirmado la existencia de variabilidad genética suficiente poder obtener respuesta a la selección. No obstante, dada la relación negativa (a más prolificidad, menor supervivencia) y la correlación genética negativa detectada entre este carácter y la superviviencia de las crías, es necesario establecer una estrategia de selección que tengan en cuenta ambos caracteres y, evidentemente, la mejora de las condiciones de explotación de las ovejas.

La existencia de sementales con valoración genética positiva para ambos caracteres asegura la posibilidad de su utilización para la mejora de aquellos ganaderos que así lo demanden. Sería muy interesante realizar otros estudios tendentes a la reducción de la variabilidada en esta variable (principalmente disminuir la tasa de partos simples y triples en favor de los dobles).

\section{AGRADECIMIENTOS}

Este trabajo ha sido financiado mediante por el Convenio de Colaboración entre la Universidad de Cór- doba y la Asociación Nacional de Criadores de raza Merina.

\section{BIBLIOGRAFÍA}

Asworth, C.J. 1992. Synchrony embryo oestrus. Anim Reprod Sci, 8: 259-267.

Foulley J.L. and Manfredi E. 1991. Approches statistiques de l'évaluation génétique des reproducteurs pour des caractères binaires à seuils. Genet Sel Evol, 23: 309-338.

Gill, J.W.; Hosking, B.J. and Egan, A.R. 1998. Prenatal programming of mammalian growth: a review of the role of steroids. Livest Prod Sci, 54: 251-267.

Gilmour, A.R.; Gogel, B.J.; Cullis, B.R. and Thompson, R. 2009. ASReml User Guide Release 3.0 VSN International Ltd. Hemel Hempstead, HP1 1ES. UK. www.vsni.co.uk.

Magrama, 2011. Programa de mejora de la raza ovina Merina, 2011. Grupo Meragem. Ministerio de Agricultura, Alimentación y Medio Ambiente. http://www.magrama.gob.es/es/ganaderia/temas/zootecnia/razas-ganaderas/razas/catalogo/autoctona-fomento/ovino/merina/

Menéndez Buxadera, A.; Alexandre, G. and Mandonnet, N. 2004. Discussion on the importance, definition and genetic components of the number of animals born in the litter with particular emphasis on small ruminants in tropical conditions. Small Ruminant Res, 54: 1-11.

Molina, A.; Medina, C.; Barajas, F.; Gómez, J. y Menéndez-Buxadera, A. 2015. Análisis de la productividad numérica y del rendimiento productivo de la raza Merina. XI Congreso de la Federación Iberoamericana de Razas Criollas y Autóctonas. 19 al 21 de marzo. Zaragoza. España. 